\title{
Metaanálisis: Efectividad del tratamiento a largo y corto plazo con albendazol para la neurocisticercosis intraparenquimatosa
}

Teodoro Edmundo Jerves Serrano ${ }^{1, a}$

RESUMEN

Objetivo: Determinar la efectividad del tratamiento con Albendazol de larga y corta duración en adultos y niños con neurocisticercosis parenquimatosa cística o transicional, verificado por neuroimagen.

Materiales y métodos: Un metaanálisis fue realizado analizando los ensayos clínicos de Medline y LILACS con un puntaje de Jadad $>$ 2. Estudios adicionales no fueron identificados en Clinicaltrials.gov y Researchgate. La variable principal fue la persistencia de las lesiones radiológicas y la secundaria los efectos adversos. El RR acumulado y un intervalo de confianza del 95 \% fueron calculados para las dos variables usando el modelo de efectos fijos de Mantel-Haenszel. El análisis fue realizado en el programa Stata 11. La heterogeneidad fue evaluada con el prueba chi cuadrado, una $p<0,10$ fue considerada estadísticamente significativa. El sesgo de publicación fue evaluado por el análisis de sensibilidad y la prueba de Egger.

Resultados: Cuatro estudios fueron incluidos. No hay diferencia entre la terapia de larga y corta duración para la neurocisticercosis. El RR acumulado de la persistencia de lesiones radiológicas después del tratamiento a corto plazo en comparación al de largo plazo fue 1,05 (IC 95 \% 0,72-1,54). La prueba de Mantel-Haenszel demostró un valor de p de 0,789. Además, ambos, el análisis de sensibilidad y la prueba de Egger apoyaron la ausencia de sesgo de publicación. No hubo diferencia significativa respecto a los efectos adversos.

Conclusiones: La evidencia sugiere que no hay diferencia entre el tratamiento de corto y largo plazo para las lesiones parenquimatosas transicionales únicas, por lo que el tratamiento de corto plazo puede ser usado efectivamente.

Palabras clave: neurocisticercosis; albendazol; efectividad; tratamiento (Fuente: DeCS BIREME).

\section{Meta-analysis: Effectiveness of long- and short-term albendazole therapy for intraparenchymal neurocysticercosis}

ABSTRACT

Objective: To determine the effectiveness of long- and short-term albendazole therapy in adults and children with cystic and transitional parenchymal neurocysticercosis confirmed by neuroimaging.

Materials and methods: A meta-analysis was performed using clinical trials from MEDLINE and LILACS with a Jadad score $>2$. Additional studies were not found on ClinicalTrials.gov and ResearchGate. The main endpoint was the persistence of radiological lesions and the secondary endpoint was the presence of adverse effects. Pooled relative risks (RR) and $95 \%$ confidence intervals were calculated for both endpoints using the Mantel-Haenszel fixed-effects model. The analysis was conducted using software Stata 11. Heterogeneity was assessed by chi-square test and a value of $p<0.10$ was considered statistically significant. Publication bias was estimated by sensitivity analysis and Egger's test.

Results: Four studies were included. There was no difference between long- and short-term albendazole therapies for neurocysticercosis. Pooled RR of the persistence of radiological lesions after short-term vs. long-term therapy was 1.05 (95 $\% \mathrm{Cl}$ 0.72-1.54). Mantel-Haenszel test showed a p-value of 0.789 . Moreover, both the sensitivity analysis and Egger's test supported the absence of publication bias. No significant difference was noticed regarding the adverse effects.

Conclusions: Evidence suggests that there is no difference between short- and long-term therapies for single transitional parenchymal lesions; therefore short-term therapy may be used effectively.

Keywords: Neurocysticercosis; albendazole; effectiveness; therapy (Source: MeSH NLM).

1. Morristown Medical Center. Nueva Jersey, Estados Unidos.

a. Doctor en Medicina, Residente de Medicina Interna. 


\section{INTRODUCCIÓN}

La neurocisticercosis (NCC) es una infección parasitaria del sistema nervioso central (SNC) por el estadio larvario de la Tenia solium, la cual ocurre cuando un humano actúa con huésped intermediario del ciclo de vida del parásito ${ }^{(1,2)}$. Esta patología es una causa común de epilepsia adquirida en adultos y niños ${ }^{(3)}$.

La NCC es una enfermedad pleomórfica, dependiendo de la localización puede dividirse en dos categorías: cerebral o parenquimatosa y extraparenquimatosa (subaracnoide, cisternal, intraventricular o espinal), según el número de lesiones es simple (una lesión) y múltiple (varias lesiones). Una subclasificación de la NCC parenquimatosa incluye el nivel de actividad o estadio de la lesión, siendo activa cuando el cisticerco está vivo, transicional en la fase degenerativa e inactiva cuando el parásito está muerto ${ }^{(4,5)}$.

La resonancia magnética (RM) y la tomografía computarizada (TC) son útiles para clasificar objetivamente la NCC determinando la localización, estadio y número de lesiones. Además, las mismas evalúan la efectividad del tratamiento demostrando la persistencia, resolución o calcificación de las lesiones ${ }^{(6)}$.

El pronóstico de la NCC ha mejorado después de la introducción de terapias sintomáticas con antiepilépticos y corticoides. Los antiparasitarios juegan un papel importante en la resolución de las lesiones activas o transicionales ${ }^{(7)}$. El albendazol posee una mejor efectividad y menos efectos adversos en comparación al praziquantel (8). Sin embargo, la duración y la dosis del tratamiento con albendazol no están definidas, con un tiempo de tratamiento que va desde los 3 a los 30 días $(8,9,10)$. La respuesta inmune del paciente, el número y estadio de las lesiones pueden modificar e influenciar la efectividad de los antiparasitarios ${ }^{(6)}$.

El último metaanálisis llevado a cabo por Abba et al en el 2010 no mostró diferencia significativa entre la terapia de larga o corta duración con albendazol para la neurocisticercosis, incluyó 6 estudios, de los cuales no fueron ensayos clínicos y otros dos tenían una baja calidad basados en un puntaje de Jadad menor de $3^{(11)}$.

Los ensayos clínicos tienen una multitud de resultados, utilizando diferentes variables, tiempo de seguimiento y duración de la terapia. Existe la incertidumbre si el tratamiento a largo plazo ( $\geq 8$ días) es mejor que el de corto plazo (<8 días) para erradicar las lesiones activas 0 transicionales de la NCC parenquimatosa ${ }^{(2,4,12)}$.

El objetivo de esta investigación es determinar si la duración del tratamiento con albendazol a largo plazo es más efectiva en comparación al tratamiento de corto plazo para la resolución radiológica de las lesiones activas o transicionales de NCC parenquimatosa en niños y adultos.

\section{MATERIALES Y MÉTODOS}

Este es un metaanálisis, cuyo protocolo fue elaborado siguiendo las recomendaciones del PRISMA-P 2015 y el reporte final según el PRISMA ${ }^{(13,14)}$.

La búsqueda en PROSPERO demostró ningún metaanálisis en proceso de ejecución. Para la identificación de los estudios se revisaron las bases de datos de Medline y LILACS sin restricción de fecha o lenguaje, buscando solo estudios en humanos. Las palabras clave usadas para la estrategia de búsqueda fueron "albendazole" AND "neurocysticercosis".

Adicionalmente, se revisó Clinicaltrials.gov y Researchgate para determinar si otros estudios existieron o están en proceso de ejecución.

Cada estudio fue codificado y analizado por autor, año de publicación siguiendo los criterios de selección y exclusión (Figura 1). Solo se incluyeron ensayos clínicos con un puntaje de Jadad $\geq 3$, excluyendo los estudios con baja calidad ${ }^{(15,16,17)}$.

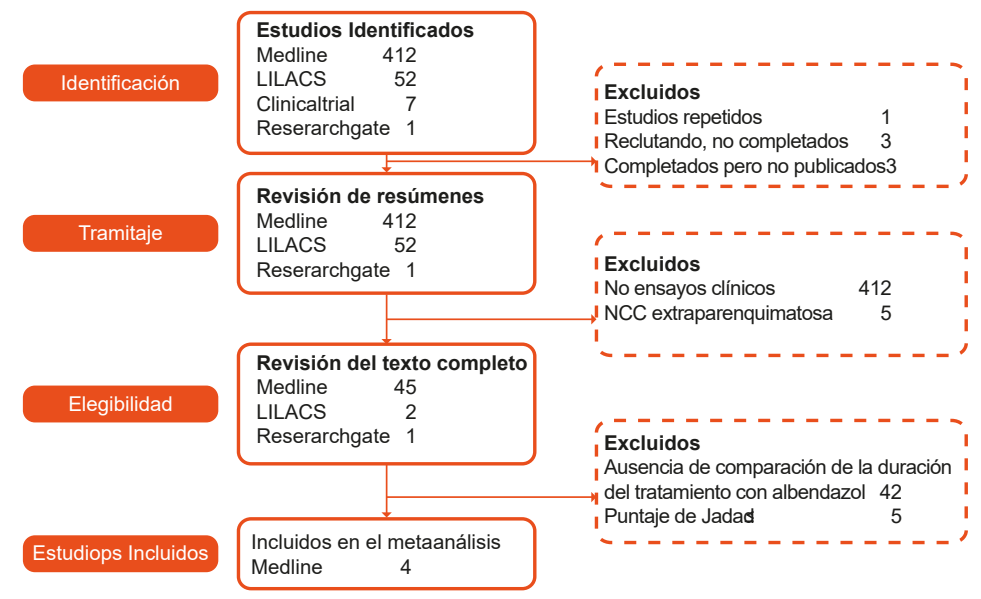

Figura 1. Criterios de selección de los estudios 
La principal variable fue la persistencia de las lesiones radiológicas después de un seguimiento de 306 meses, siendo esta categórica. La variable secundaria fue la presencia de efectos adversos. Un subconjunto de datos fue analizado para determinar la variación de los resultados considerando las calcificaciones como persistencia o no de las lesiones y según el grupo de edad. El programa Stata 11 fue utilizado para realizar el análisis estadístico. La heterogeneidad entre estudios fue evaluada usando la prueba de chi cuadrado, una $p<0,10$ fue considerada estadísticamente significativa en el análisis de heterogeneidad. Los riesgos relativos (RR) acumulados y el $95 \%$ de intervalo de confianza (IC $95 \%$ ) se emplearon para las variables principal y secundaria. Los cálculos se realizaron usando el modelo de efectos fijos de Mantel-Haenszel en la ausencia de heterogeneidad. Los RR acumulados fueron calculados dividiendo la incidencia de lesiones persistentes en el tratamiento a corto plazo para la incidencia de lesiones en el tratamiento a largo plazo. El sesgo de publicación fue valorado por la prueba de Egger y el análisis de sensibilidad.

\section{RESULTADOS}

Cuatro estudios fueron considerados en el metaanálsis. La metodología de los estudios incluidos fue similar, todos los estudios utilizaron antiepilépticos y fueron aleatorizados. La mayor diferencia fue que los estudios de de Khurana et al y Kaur et al no fueron cegados, mientras que los estudios de Singhi et al y Garcia et al lo fueron. Khurana usó la RM para evaluar la persistencia de las lesiones y no uso corticoides como tratamiento adicional. Solo adultos participaron en el estudio de García et al, mientras que niños y adultos fueron incluidos en los otros tres estudios ${ }^{(18-21)}$.

Las principales diferencias y características de los estudios (Tabla 1).

Tabla 1. Principales características de los ensayos clínicos

$\begin{array}{ccccc}\begin{array}{c}\text { Autores y año de } \\ \text { publicación }\end{array} & \text { Garcia et al. (1997) } & \text { Singhi et al. (2003) } & \text { Kaur et al. (2010) } & \text { Khurana et al. (2012) } \\ \text { Seguimiento (meses) } & 3 & 3-6 & 3-6 & 6 \\ \text { Grupo de edad } & \text { Adultos } & \text { Niños } & \text { Niños } & \text { Adultos/ Niños } \\ \text { Cegamiento } & \text { Doble ciego } & \text { Doble ciego } & \text { Abierto } & \text { Abierto } \\ \text { Pérdida de seguimiento } & 5 & 25 & 9 & 0 \\ \text { Tipo de análisis } & \text { Por protocolo } & \text { Por protocolo } & \text { Intención de tratar } & \text { Por protocolo } \\ \text { Dosis del albendazol } & 400 \mathrm{mg} \mathrm{BID} & 15 \mathrm{mg} / \mathrm{kg} / \mathrm{día} & 15 \mathrm{mg} / \mathrm{kg} / \mathrm{día} & 15 \mathrm{mg} / \mathrm{kg} / \mathrm{d} \text { ía } \\ \text { Tiempo de tratamiento (días) } & 7 \text { vs } 14 & 7 \mathrm{vs} 28 & 7 \mathrm{vs} 28 & 3 \text { vs } 15 \\ \text { Corticoides } & \mathrm{Si} & \mathrm{Si} & \mathrm{Si} & \text { No } \\ \text { Método de neuroimagen } & \text { TC } & \text { TC } & \text { TC } & \text { RM } \\ \text { Estadio de la lesión } & \text { Quiste / Transicional } & \text { Transicional } & \text { Transicional } & \text { Transicional } \\ \text { Número de lesiones } & 1 \text { y múltiples } & 1 \text { a 3 } & 1 & 1 \\ \text { Puntaje de Jadad } & 4 & 4 & 3 & 3\end{array}$

El total de pacientes fue 321, distribuidos en 160 en el grupo de tratamiento a corto plazo y 161 en el tratamiento a largo plazo. El tamaño de la muestra fue similar en todos los estudios lo que refleja la ausencia de heterogeneidad significativa. En las tablas 2 y 3 demuestran la persistencia de las lesiones en cada estudio a los 3 y 6 meses de seguimiento respectivamente.

Tabla 2. Persistencia de las lesiones de neurocisticercosis 3 meses después de la terapia

\begin{tabular}{ccccccccc}
$\begin{array}{c}\text { Autores y año de } \\
\text { publicación }\end{array}$ & \multicolumn{3}{c}{$\begin{array}{c}\text { Persistencia de las lesions de NCC* (Calcificaciones } \\
\text { consideradas lesión persistente) }\end{array}$} & \multicolumn{5}{c}{$\begin{array}{c}\text { Persistencia de las lesions de NCC* (Calcificaciones } \\
\text { consideradas lesión resuelta) }\end{array}$} \\
& \multicolumn{2}{c}{$\begin{array}{c}\text { Terapia a corto plazo } \\
\text { Sí }\end{array}$} & No & Terapia a largo plazo & Terapia a corto plazo & \multicolumn{2}{c}{ Terapia a largo plazo } \\
Kaur et al. (2010) & 21 & 37 & 30 & 32 & 15 & 43 & 15 & 47 \\
Singhi et al. (2003) & 13 & 49 & 14 & 46 & 7 & 55 & 9 & 51 \\
Garcia et al. (1997)+ & - & - & - & - & 4 & 1 & 3 & 1
\end{tabular}

${ }^{*} \mathrm{NCC}=$ neurocisticersosis

+Solamente los pacientes con lesions simples transicionales parenquimatosas fueron incluidos 
Metaanálisis: Efectividad del tratamiento a largo y corto plazo con albendazol para la neurocisticercosis intraparenquimatosa

Tabla 3. Persistencia de las lesiones de neurocisticercosis 6 meses después de la terapia

\begin{tabular}{|c|c|c|c|c|c|c|c|c|}
\hline \multirow[t]{3}{*}{$\begin{array}{l}\text { Autores y año de } \\
\text { publicación }\end{array}$} & \multicolumn{4}{|c|}{$\begin{array}{l}\text { Persistencia de las lesions de NCC* (Calcificaciones } \\
\text { consideradas lesión persistente) }\end{array}$} & \multicolumn{4}{|c|}{$\begin{array}{c}\text { Persistencia de las lesions de NCC* (Calcificaciones } \\
\text { consideradas lesión resuelta) }\end{array}$} \\
\hline & \multicolumn{2}{|c|}{ Terapia a corto plazo } & \multicolumn{2}{|c|}{ Terapia a largo plazo } & \multicolumn{2}{|c|}{ Terapia a corto plazo } & \multicolumn{2}{|c|}{ Terapia a largo plazo } \\
\hline & Sí & No & Sí & No & Sí & No & Sí & No \\
\hline Khurana et al. (2012) (29) & 16 & 19 & 11 & 24 & 13 & 22 & 10 & 25 \\
\hline Kaur et al. (2010) (28) & 21 & 37 & 30 & 32 & 15 & 43 & 15 & 47 \\
\hline Singhi et al. (2003) (30) & 11 & 51 & 8 & 52 & 5 & 57 & 2 & 58 \\
\hline
\end{tabular}

${ }^{*} \mathrm{NCC}=$ neurocisticersosis

Los resultados respaldan que no existe diferencia estadísticamente significativa de la duración de la terapia para neurocisticercosis parenquimatosa simple transicional. El RR acumulado de la persistencia de lesiones comparando el tratamiento de largo y corto plazo fue 1,05 (IC95 \% 0,721,54) (Figura 2) y el valor de $\mathrm{p}$ de Mantel-Haenszel fue 0.789. Tomando en cuenta el sesgo de publicación a través de la prueba de Egger $(p=0,291)$ y el análisis de sensibilidad (Figura 3), sugieren que no hay sesgo de publicación. El patrón es el mismo indistintamente del grupo de edad, tiempo de resolución y el hecho de si las calcificaciones son consideradas como lesiones persistentes o resueltas tampoco cambia los resultados.

\begin{tabular}{|c|c|c|c|}
\hline Estudio & & RR (IC 95\%) & $\%$ Peso \\
\hline & i & & \\
\hline Kaur et al. (2010) & $\begin{array}{l}1 \\
\text { I } \\
\text { i }\end{array}$ & $1,07(0,58-1,99)$ & 39,21 \\
\hline Singhi et al. (2003) & $\begin{array}{l}\text { I } \\
\text { I } \\
\text { I }\end{array}$ & $0,75(0,30-1,89)$ & 24,74 \\
\hline Garcia et al. (1997) & $\begin{array}{l}1 \\
1 \\
1\end{array}$ & $1,07(0,52-2,18) 9,01$ & 9,01 \\
\hline Khurana et al. (2012) & $\frac{1}{1}$ & $1,30(0,66-2,56) 27,04$ & 27,04 \\
\hline Global $(p=0.829)$ & I & $1,05(0,72-1,54) 100,00$ & 100,00 \\
\hline 1 & & 1 & \\
\hline 0.2 & 1 & 3 & \\
\hline
\end{tabular}

Figura 2. Persistencia de las lesiones simples transicionales de neurocisticercosis a los 3 y 6 meses después del tratamiento a corto plazo en comparación con el tratamiento a largo plazo con albendazol 


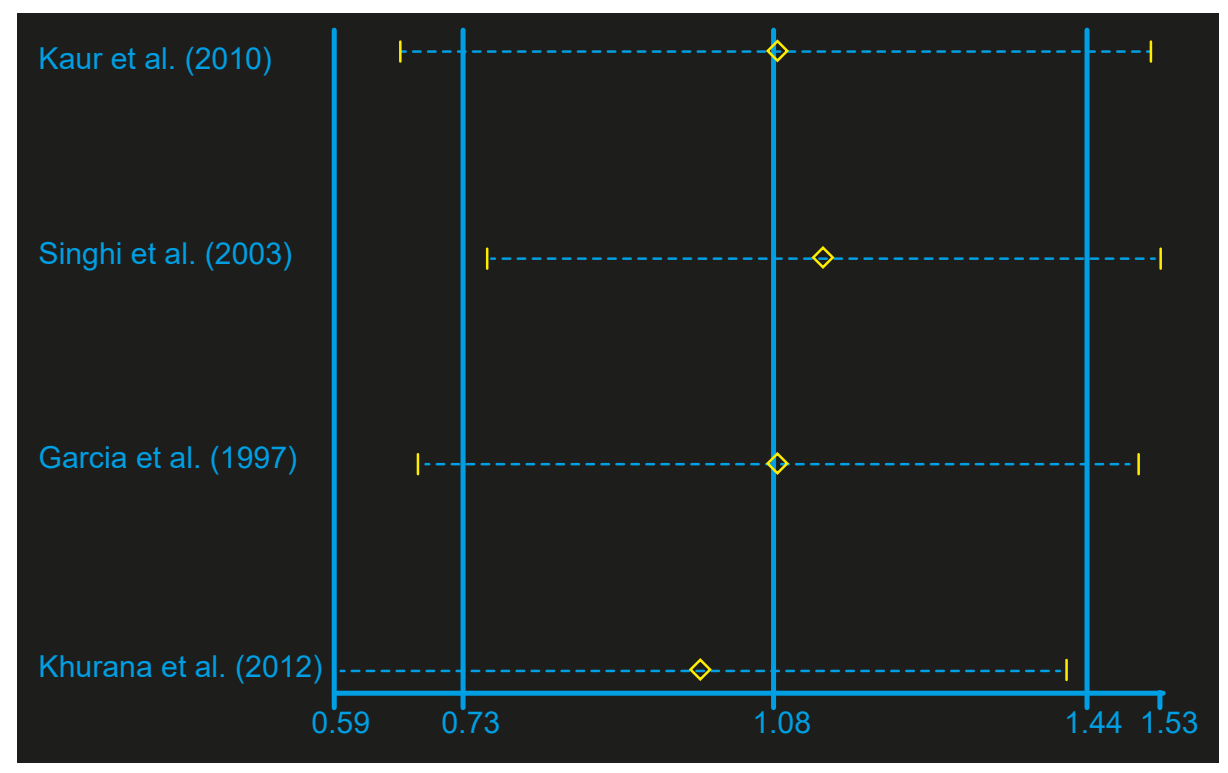

Figura 3. Análisis de sensibilidad

Solamente los efectos adversos gastrointestinales fueron considerados importantes. No hubo diferencia significativa en los de efectos adversos del tratamiento a largo plazo en comparación al de corto plazo, el RR acumulado fue 0,55 (IC $95 \%$ 0,17 - 1,79).

\section{DISCUSIÓN}

Más estudios se necesitan para obtener conclusiones acerca de la efectividad de la duración tratamiento en el caso de lesiones múltiples y cuando el estadio de la lesión es quiste o viable. La mayoría de los estudios solo analiza lesiones simple transicionales, complicando la generalización de los resultados encontrados a lesiones múltiples.

El último metaanálisis fue llevado a cabo por Abba et al en el 2010 mostrando que no existe diferencia significativa entre el tratamiento con albendazol a largo o corto plazo para la neurocisticercosis. El RR acumulado de la persistencia de las lesiones comparando el tratamiento a largo y corto plazo fue 0,98 (IC95\% 0,64 - 1,5) y el valor de p de Mantel-Haenszel fue 0,92. Los resultados concuerdan con el actual metaanálisis. Las principales diferencias entre estos estudios radican en que solo los ensayos clínicos de alta calidad fueron incluidos en el estudio actual.

La NCC se ha convertido en un problema de salud tanto en los países desarrollados como en desarrollo. Un mundo globalizado ha acortado distancias entre los países lo cual facilita la transmisión de NCC, incrementando la

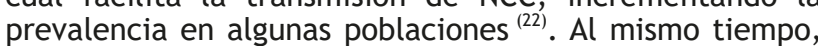
la difusión de conocimiento y terapias disminuyen la prevalencia en otras poblaciones ${ }^{(23)}$.

La complejidad de la NCC explica algunas barreras para definir la mejor dosis y duración del tratamiento. El número de lesiones puede ir de uno a cientos. Aún más, una lesión puede producir convulsiones y muchas pueden ser asintomáticas. De la misma manera, los síntomas de las lesiones parenquimatosas dependen en la localización y área cerebral afectada.6 Así que, múltiples enfermedades neurológicas pueden ser simuladas por la NCC. Las lesiones extraparenquimatosas tienen peor pronóstico y mayor tendencia a necesitar cirugía ${ }^{(24)}$.

El manejo de esta enfermedad infecciosa que se disemina por vía fecal-oral debe incluir medidas preventivas como la higiene de manos acompañada de un tratamiento adecuado para prevenir la diseminación de la enfermedad y reinfección ${ }^{(1)}$. Una evaluación inicial de cada paciente tomando en cuenta el estado de las lesiones, localización y recurrencia se debe llevar a cabo antes de iniciar el tratamiento y frecuencia y dosis ${ }^{(22)}$.

Aunque los corticoides y antiepilépticos son usados frecuentemente en el manejo de la NCC, problemas sobre la dosis, tipo y tiempo todavía son debatidos (25). El praziquantel es una opción de tratamiento de segunda línea después de que un metaanálisis demostró una mejor efectividad del albendazol ${ }^{(26)}$. La terapia con $400 \mathrm{mg}$ de albendazol dos veces por día en adultos y $15 \mathrm{mg} / \mathrm{kg} /$ día dos veces por día en los niños es la dosis más común ${ }^{(18,19,20,21)}$.

La terapia combinada abre las puertas a la discusión entre expertos. Un estudio elaborado por García et al incrementó la dosis del albendazol a $22,5 \mathrm{mg} /$ $\mathrm{kg} /$ día y basado en estudio in vitro que sugieren un sinergismo, combinó las terapias de albendazol y praziquantel obteniendo una mejor eficacia con la terapia combinada sin incrementar significativamente los efectos adversos ${ }^{(27)}$. 
Los resultados del actual metaanálisis apoyan el uso del tratamiento de corta duración (menos de 8 días) con albendazol en la neurocisticercosis parenquimatosa simple transicional en adultos y niños.

Este argumento se basa en la misma efectividad de la duración de los tratamientos. A pesar de que los efectos adversos no incrementaron significativamente, se necesitan más para respaldar este resultado. Pues el tratamiento a largo plazo incrementa los costos y quizás los efectos adversos sin una efectividad significativa.

Algunos de los inconvenientes identificados después de completar el metaanálisis fueron el hecho de considerar a la persistencia de las lesiones como variable principal ${ }^{(28)}$. La mayoría de los expertos concuerdan que la efectividad del tratamiento debe enfocarse en la recurrencia de los signos y síntomas, principalmente las convulsiones ${ }^{(29)}$. Sin embargo, una limitada cantidad de estudios consideran a la recurrencia de convulsiones como la variable principal ${ }^{(30,31)}$.

Más ensayos clínicos de alta calidad con una metodología mejorada son necesarios para responder las preguntas que quedan. Un poder adecuado es otro punto a considerar en los nuevos estudios. Además, el reporte de los efectos adversos debe ser más claro.

La respuesta inmune y los factores genéticos son menos entendidos como covariables en la ecuación. El desarrollo de estas áreas en los siguientes años puede producir información valiosa para descifrar la complejidad de esta patología y la variabilidad en la respuesta al tratamiento ${ }^{(6)}$.

Los resultados del estudio Effects of 2 different duration of albendazole therapy in patients with neurocysticercosis in brain $\leq 5$ lesions on CT serán útiles para clarificar algunas de las interrogantes. El estudio se completó pero su publicación está pendiente según el portal de clinicaltrials.gov.

Aunque, no hay diferencia entre el tratamiento a corto y largo plazo con albendazol, algunos puntos no están claros. Por ejemplo, se necesitan más estudios para establecer si 3 días de tratamiento tienen la misma efectividad en comparación a 7. La efectividad de una dosis mayor de albendazol o la terapia combinada con praziquantel deben ser analizadas.

Sin embargo, estudios con alta calidad como el caso de los ensayos clínicos aleatorizados se necesitan para obtener conclusiones acerca de estos puntos.

\section{REFERENCIAS BIBLIOGRÁFICAS}

1. Takayanagui OM. Neurocysticercosis. Arq Neuropsiquiatr. 2013;71(9B):710-713. Disponible en: http://www.scielo.br/ pdf/anp/v71n9B/0004-282X-anp-71-09b-710.pdf

2. Garcia $\mathrm{HH}$, Nash TE, Del Brutto $\mathrm{OH}$. Clinical symptoms, diagnosis, and treatment of neurocysticercosis. Lancet Neurol. 2014;13(12):1202-15. Disponible en: https://www. sciencedirect.com/science/article/pii/S1474442214700948
3. Ocana GS, Sablon JCO, Tamayo IO, Arena LA, Ocana LMS, Govender S. Neurocysticercosis in patients presenting with epilepsy at St Elizabeth's Hospital, Lusikisiki. S Afr Med J. 2009;99(8):588-91. Disponible en: http://www.scielo.org. za/scielo. php?pid=S0256-95742009000800017\&script=sci arttext\&tlng=en

4. Otte W, Singla M, Sander J, Singh G. Drug therapy for solitary cysticercus granuloma: a systematic review and metaanalysis. Neurology. 2013; 80(2):152-62. Disponible en: http://n.neurology.org/content/80/2/152. short

5. Saavedra H, Gonzales I, Alvarado MA, Porras MA, Vargas V, Cjuno RA, et al. Diagnóstico y manejo de la neurocisticercosis en el Perú. Rev Peru Med Exp Salud Pública. 2010;27(4):58691. Disponible en: http://www.scielo.org.pe/scielo. php?pid=S1726-46342010000400015\&script=sci_arttext

6. Carpio A, Fleury A, Hauser W. Neurocysticercosis: Five new things. Neurol Clin Pract. 2013; 3(2): 118-125. Disponible en: http://cp. neurology.org/content/3/2/118. short

7. Nogales-Gaete J, Arriagada C, Salinas R. Tratamiento de la neurocisticercosis: Revisión crítica. Rev Méd Chile. 2006;134(6):789-96. Disponible en: https://scielo.conicyt. $\mathrm{cl} /$ scielo.php?pid=S0034-98872006000600017\&script=sci_ arttext\&tlng=pt

8. Matthaiou D, Panos G, Adamidi E, Falagas M. Albendazole versus Praziquantel in the Treatment of Neurocysticercosis: A Meta-analysis of Comparative Trials. PLoS Negl Trop Dis. 2008; 2(3):e194. Disponible en: http://journals.plos.org/plosntds/ article?id=10.1371/journal.pntd.0000194

9. Chaurasia RN, Garg RK, Agarwal A, Kohli N, Verma R, Singh MK, et al. Three day albendazole therapy in patients with a solitary cysticercus granuloma: a randomized double blind placebo controlled study. Southeast Asian J Trop Med Public Health. 2010;41(3):517-25. Disponible en: https://search. proquest.com/docview/578162396?pq-origsite=gscholar

10. Sotelo J, Del Brutto O, Penagos P, Escobedo F, Torres B, Rodriguez-Carbajal J, et al. Comparison of therapeutic regimen of anticysticercal drugs for parenchymal brain cysticercosis. J Neurol. 1990;237(2):69-72. Disponible en: https: / / link.springer.com/article/10.1007/BF00314663

11. Abba K, Ramaratnam S, Ranganathan LN. Anthelmintics for people with neurocysticercosis. Cochrane Database Syst Rev. 2010;20 (1). Disponible en: https://www.ncbi.nlm.nih.gov/ pubmed/20091504

12. Bustos J, Pretell E, Llanos-Zavalaga F, Gilmanb R, Del Brutto O, Garcia H. Efficacy of a 3-day course of albendazole treatment in patients with a single neurocysticercosis cyst. Clinical Neurology and Neurosurgery. 2006; 108(2):193-194. Disponible en: https://jhu.pure.elsevier.com/en/publications/efficacyof-a-3-day-course-of-albendazole-treatment-in-patients-w-4

13. Moher D, Shamseer L, Clarke M, Ghersi D, Liberati A, Petticrew $M$, et al. Preferred reporting items for systematic review and meta-analysis protocols (PRISMA-P) 2015 statement. Syst Rev. 2015;4(1):1. Disponible en: https://www.ncbi.nlm.nih.gov/ pubmed $/ 25554246$

14. Moher D, Liberati A, Tetzlaff J, Altman DG, Group P. Preferred reporting items for systematic reviews and meta-analyses: the PRISMA statement. PLoS Med. 2009;6(7):e1000097. Disponible en: https://www.ncbi.nlm.nih.gov/pubmed/19621072

15. Moher D, Jadad AR, Tugwell P. Assessing the quality of randomized controlled trials: current issues and future directions. Int J Technol Assess Health Care. 1996;12(2):195208. Disponible en: https://www.ncbi.nlm.nih.gov/ pubmed/8707495

16. Cruz I, Cruz M, Carrasco F, Horton J. Neurocysticercosis: optimal dose treatment with albendazole. J Neurol Sci. 1995;133(1):152-4. Disponible en: https://www.ncbi.nlm.nih. gov/pubmed/8583218 
17. Alarcón F, Dueñas G, Diaz M, Cevallos N, Estrada G. Short course of albendazole therapy for neurocysticercosis: A prospective randomized trial comparing three days, eight days and the control group without albendazole. Rev Ecuat de Neurol. 2000; 10(1):1-6. Disponible en: http:// revecuatneurol.com/magazine_issue_article/albendazoletherapy-neurocysticercosis-randomized-trial/

18. Kaur P, Dhiman P, Dhawan N, Nijhawan R, Pandit S. Comparison of 1 week versus 4 weeks of albendazole therapy in single small enhancing computed tomography lesion. Neurol India. 2010; 58(4): 560-564. Disponible en: https://www.ncbi.nlm. nih.gov/pubmed/20739792

19. Khurana N, Garg R, Verma R, Malhotra H, Singh M, Shukla R. Three-day versus 15-day course of albendazole therapy in solitary cysticercus granuloma: an open label randomized trial. J Neurol Sci. 2012; 316(1-2):36-41. Disponible en: https://www.ncbi.nlm.nih.gov/pubmed/22342396

20. Singhi $P$, Dayal D, Khandelwal N. One week versus four weeks of albendazole therapy for neurocysticercosis in children: a randomized, placebo-controlled double blind trial. Pediatr Infect Dis J. 2003; 22(3): 268-72. Disponible: https://www. ncbi.nlm.nih.gov/pubmed/12634590

21. Garcia H, Gilman R, Horton J, Martinez M, Herrera G, Altamirano J, et al. Albendazole therapy for neurocysticercosis A prospective double-blind trial comparing 7 versus 14 days of treatment. Neurology. 1997;48(5):1421-1427. Disponible en: http://n.neurology.org/content/48/5/1421.short

22. Del Brutto O. Neurocysticercosis: A Review. Scientific World Journal. 2012. Disponible en: https://www.ncbi.nlm.nih.gov/ pmc/articles/PMC3261519/

23. Esquicha JA, Falcón N, Oshiro S. Características clínicas y epidemiológicas de los pacientes con neurocisticercosis en un hospital general de Lima. Rev Med Hered. 2012;23(1):410. Disponible en: http://www.scielo.org.pe/scielo. php?pid=S1018-130X2012000100003\&script=sci_arttext

24. Göngora-Rivera F, Soto-Hernández J, Esquivel DG, Cook H, Márquez-Caraveo $\mathrm{C}$, Dávila $\mathrm{RH}$, et al. Albendazole trial at 15 or $30 \mathrm{mg} / \mathrm{kg} /$ day for subarachnoid and intraventricular cysticercosis. Neurology. 2006;66(3):436-8. Disponible en: http://n. neurology.org/content/66/3/436.short

25. Singh G, Rajshekhar V, Murthy J, Prabhakar S, Modi M, Khandelwal $\mathrm{N}$, et al. A diagnostic and therapeutic scheme for a solitary cysticercus granuloma. Neurology. 2010; 75(24): 2236-2245. Disponible en: http://n.neurology.org/ content/75/24/2236.short

26. Del Brutto O, Roos K, Coffey C, García H. Meta-Analysis: Cysticidal Drugs for Neurocysticercosis: Albendazole and Praziquantel. Ann Intern Med. 2006; 145(1):43-51. Disponible en: https://www.ncbi.nlm.nih.gov/pubmed/16818928

27. Garcia H, Gonzales I, Lescano A, Bustos J, Zimic M, Escalante $D$, et al. Efficacy of combined antiparasitic therapy with praziquantel and albendazole for neurocysticercosis: a double-blind, randomised controlled trial. Lancet Infect Dis. 2014; 14(8): 687-95. Disponible en: https://www.ncbi.nlm. nih.gov/pmc/articles/PMC4157934/

28. Carpio A, Kelvin E, Bagiella E, Leslie D, Leon P, Andrews H, et al. Effects of albendazole treatment on neurocysticercosis: a randomised controlled trial. J Neurol Neurosurg Psychiatry. 2008; 79(9): 1050-1055. Disponible en: https://www.ncbi. $\mathrm{nlm}$.nih.gov/pubmed/18495737

29. Garcia H, Javier Pretell E, Gilman R, Martinez S, Moulton L. A Trial of Antiparasitic Treatment to Reduce the Rate of Seizures Due to Cerebral Cysticercosis. N Engl J Med. 2004; 350(3): 249-58. Disponible en: https://www.ncbi.nlm.nih. gov/pubmed/14724304

30. Romo M, Wyka K, Carpio A, Leslie D, Andrews H, Bagiella E, et al. The effect of albendazole treatment on seizure outcomes in patients with symptomatic neurocysticercosis. Trans R Soc Trop Med Hyg. 2015; 109(11): 738-46. Disponible en: https:// www.ncbi.nlm.nih.gov/pubmed/26433183

31. Das K, Mondal G, Banerjee M, Mukherjee B, Singh O. Role of antiparasitic therapy for seizures and resolution of lesions in neurocysticercosis patients: An 8 year randomised study. J Clin Neurosci. 2007; 14(12): 1172-1177. Disponible en: https://www.sciencedirect.com/science/article/pii/ S0967586807000069

\section{Fuentes de financiamiento:}

Este artículo ha sido financiado por los autores.

\section{Conflictos de interés:}

Los autores declaran no tener ningún conflicto de interés.

\section{Correspondencia:}

Teodoro Edmundo Jerves Serrano

Dirección: 100 Franklin street departamento 308 edificio $\mathrm{H}$ Morristown. Nueva Jersey, Estados Unidos. Código postal 07960. Teléfono: +1 5512639897

Correo electrónico: mdteojs11@gmail.com

Recibido: 02 de enero de 2018 Evaluado: 04 de enero de 2018 Aprobado: 31 de enero de 2018

(c) La revista. Publicado por Universidad de San Martín de Porres, Perú. (cc) $\mathbf{\text { в }}$ Licencia de Creative Commons Artículo en acceso abierto bajo términos de Licencia Creative Commons Atribución 4.0 Internacional. (http://creativecommons.org/licenses/by/4.0/)

ORCID iDs

Teodoro Edmundo Jerves Serrano (iD https: / / orcid.org/0000-0002-5279-3298 